\title{
ARTICLE
}

\section{Why Do Older Sprinters Reach the Finish Line Later?}

\author{
Adamantios Arampatzis ${ }^{1}$, Hans Degens ${ }^{2}$, Vasilios Baltzopoulos ${ }^{2}$, and Jörn Rittweger ${ }^{2,3}$ \\ ${ }^{1}$ Department of Training and Movement Sciences, Humboldt-University Berlin, Germany; ${ }^{2}$ Institute for \\ Biomedical Research into Human Movement and Health, Manchester Metropolitan University, United Kingdom; \\ and ${ }^{3}$ Institute of Aerospace Medicine, German Aerospace Center, Cologne, Germany
}

ARAMPATZIS, A., H. DEGENS, V. BALTZOPOULOS, and J. RITTWEGER. Why do older sprinters reach the finish line later? Exerc. Sport Sci. Rev., Vol. 39, No. 1, pp. 18-22, 2011. Slowing of movement with aging is inevitable. The mechanisms responsible for this deterioration, however, remain poorly understood. Changes to physiological and mechanical factors controlling muscle force and power production seem likely contributors, but a reduced ability for fast transmission of the force to the skeleton also may contribute to the deterioration in performance. Key Words: running, master athletes, aging, slowing, muscle, power

\section{INTRODUCTION}

It is evident that people slow down as they age. In sports, this slowing process commences around the age of $25 \mathrm{yr}$. It is interesting to note where the gradual decline, for example, in running speed, is extremely similar across various groups, to the point that one can even adjust running performance for age and thus compare running times from athletes of different ages. It seems that the age-related deterioration in running speed is more severe in long distance than in sprint events (4), and the same is true for swimming (10). However, the relationship between speed and physiological power in human locomotion is affected by drag and accumulation of kinetic energy, and these factors constitute nonlinear terms in the power equations.

Consequently, the age-related decline in running power appears to be more or less linear, and, quite strikingly, it is very similar in relative terms between distance and sprint running (29). Human power generation in other sports, such as cycling (Fig. 1), undergoes an age-related deterioration as well, and the decline is very similar to that in running. Given the very different physiological and biomechanical character-

Address for correspondence: Adamantios Arampatzis, Ph.D., Humboldt-Universität zu Berlin, Department of Training and Movement Sciences, Centre of Sport Science and Sport Medicine Berlin, Philippstr. 13, Haus 11, 10115 Berlin

(E-mail: a.arampatzis@hu-berlin.de).

Accepted for publication: September 16, 2010.

Associate Editor: Scott J. Montain, Ph.D., FACSM

0091-6331/3901/18-22

Exercise and Sport Sciences Reviews

Copyright (C) 2010 by the American College of Sports Medicine istics of such different sports, these observations give rise to the intriguing idea that age-related slowing in human locomotion may be caused by some generic factors that affect speed of movement. Thus, the question arises: which physiological or biomechanical factors are likely to be involved in this slowing process, and what are their possible interactions?

Master sprinters preserve their stride frequency, so the reduction in maximum running velocity is caused mainly by the reduction in stride length (because velocity is the product of stride length and frequency), and this in turn is caused by the reduced braking and propulsive ground reaction forces and their rate of development (14). The main physiological reasons for this are the type II fiber atrophy and the reduced maximum force and rate of force development capacity in master sprinters. A reduced elastic energy recovery in older runners also may be implicated in the reduced rate of force development, especially during the propulsive concentric phase (5).

These age-associated changes in sprinting ability might be a consequence of reduced training overload. Many master athletes participating in competitive sports report that they reduce their training frequency and duration primarily because they require more time for recovery and regeneration. Interestingly, a previous study in runners suggests that younger and older endurance runners who devote similar amounts of time to their training have equally good running performance (26). However, the reduction in training volume, generally speaking, is very moderate. It is interesting in this context that problems in the Achilles tendon (20), which are referred to as overuse injuries, do not seem to become more prevalent with old age in master athletes. Taken together, these observations probably suggest that master athletes reduce their training to some extent as they age, mainly to prevent injuries. 


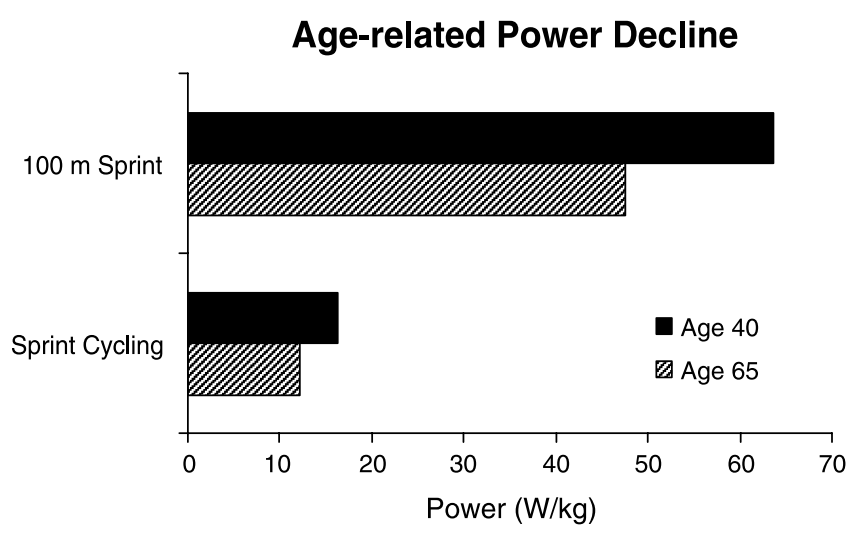

Figure 1. Comparison of the age-related decline in cycling and sprinting power. The cyclists' data were assessed as anaerobic peak power in a laboratory setting in male master cyclists (data from Martin et al. J. Gerontol. A Biol. Sci. Med. Sci. 2000;55: M311). Sprinting power has been computed according to established power equations on the basis of running world record data (data from Rittweger et al. Proc. Biol. Sci. 2009; 276:683-9) for the $100-\mathrm{m}$ sprint. The most striking feature in this diagram is that the percentage decline is very similar across the different events of sprint cycling and sprint running $(-25.3 \%$ vs $-25.4 \%$, respectively). When comparing absolute values, one must consider that cycling power in this diagram is given as external power and running power as internal (or metabolic) power. Assuming an efficiency of $\eta=0.25$ for cycling power, and thus multiplication by a factor of 4 , would yield very comparable numbers for sprint cycling and running power.

Another factor that might contribute to age-related slowing could be a change in body composition. Clearly, skeletal muscle is the generator of mechanical power in humans, and increased fat deposits, as often observed in older people, can therefore slow locomotion down. However, master athletes show virtually no change in body composition with age $(10,26)$, particularly when a high-level training regimen is maintained (27).

The main factors affecting propulsion during the contact phase of running and, ultimately, the running speed are the maximum strength, the rate of force development, and the elastic energy reuse (Fig. 2), all of which are known to deteriorate with age from previous work on master runners.

These factors are affected in turn by the changes in muscle mass, composition and architecture and in muscletendon mechanical properties, in addition to the reduced activation, control, and coordination of the neuromuscular system with aging.

The objective of this review is to examine the evidence for changes in muscle and tendon with age so as to formulate a tentative explanation for the deterioration in sprinting ability as athletes age. Our work with master athletes suggests that the primary causes of the reduced performance are the deterioration of muscle force generation and the compromised ability for fast development and transmission of the force to the skeleton. We focus on sprint running and the related muscle and tendon physiological and biomechanical issues, both in an attempt to understand human athletic performance in old age and to gain novel insights into age-related frailty.

\section{Age-Related Changes in Muscle Contractile Properties, Architecture, and Size}

Skeletal muscle contributes to the generation of power required for sprinting, and the force produced when a muscle is maximally activated varies with the velocity of shortening according to the Hill equation (13). Examples of the force-velocity

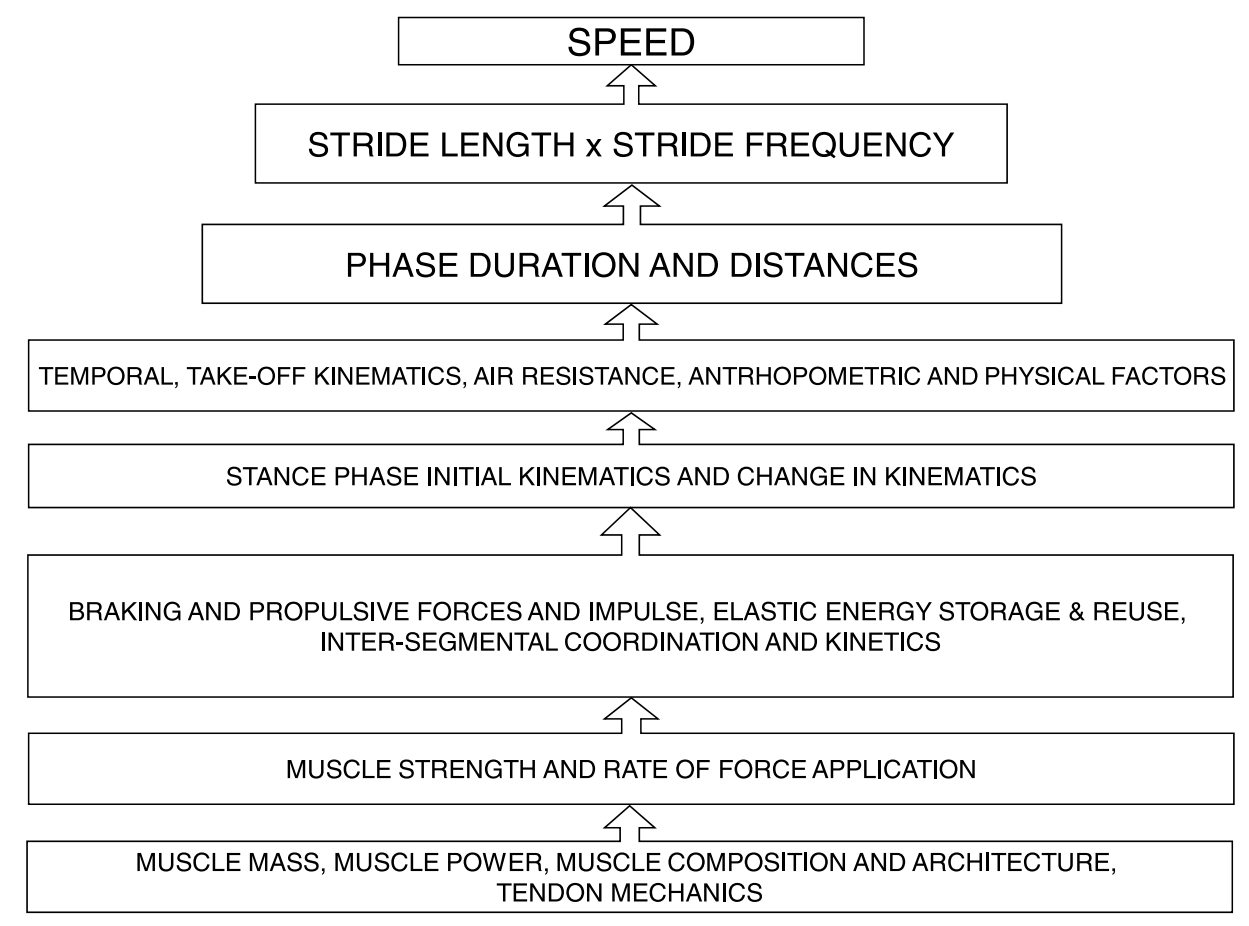

Figure 2. Simplified conceptual model relating sprinting speed to the kinematic and kinetic parameters and their changes during the stance phase that are in turn affected by the muscle force and rate of force application capacity of the athlete at a more basic level of this hierarchical model. These parameters are determined by the physiological and biomechanical characteristics of the muscles and tendon and their interaction during this dynamic activity. These fundamental properties that ultimately determine running speed are examined in this article with a particular focus on the effects of aging. 
relationship are shown in Figure 3. Power is the product of force and velocity, and it appears that peak power is produced at around $30 \%$ of the maximal velocity of shortening $\left(V_{\max }\right)$. Fibers of different myosin heavy-chain $(\mathrm{MHC}) \mathrm{com}-$ position differ in their $V_{\max }$. Our recent work (11) and previous research $(6,7,14,16)$ show that fibers consisting primarily of type II MHC have a higher $V_{\max }$ than type I fibers, but there is little difference in the maximal tetanic force per fiber cross-sectional area. It readily can be seen in Figure 3 that as a consequence of this difference in $V_{\max }$, the fast type II fibers produce markedly more power, and at higher velocities of shortening, than type I fibers. For a sprint athlete, it is thus desirable to have a large proportion of fast type II fibers.

Part of the age-related slowing might be caused by the preferential loss and/or atrophy of fast fibers during the aging process $(15,17)$, although not all studies report this (1). In Figure 3, it can be seen that the effect of this fast-to-slow transition would be twofold: 1) a reduction in the maximal power the muscle can generate and 2) a reduction in the shortening velocity at which the muscle produces maximal power. Both effects would cause a reduction in the running speed. Even in master athletes, there is an increased proportion of type I fibers and MHC, which slow muscle contractile properties (14), as well as a progressive reduction in powergenerating capacity (25). The shift in fiber-type composition might be caused by a loss of large motoneurons that innervate type II fibers (18) but also by changes in the systemic environment of the muscle cell leading to wasting and muscle fiber dysfunction. (8). In line with this, it has been shown that type I fibers of old rats are approximately $40 \%$ slower than those fibers in young-adult rats, independent of changes in the myosin light-chain composition (7). A similar situation has been reported in humans where not only type I, but also type Ila fibers have reduced maximal unloaded shortening velocities $(6,16)$. Although this is not a universal observation (34), discrepancies in the literature might be related to differences in activity levels or the type of activity. Indeed, one study showed that, although sedentary elderly had slower type I and Ila fibers, this was not the case in endurance-trained elderly people (6); another study showed that the slowing of type I fibers was not prevented in sprint master athletes (14).

Part of the slowing of contractile properties in old age might be caused by the glycation of myofibrillar proteins. In support of this, the velocity of movement of actin filaments in in vitro motility assays was reversibly reduced when the myosins were incubated in glucose (28). Such a situation might occur with development of insulin resistance and as the result of oxidative stress caused by impaired mitochondrial function (24). The muscles from old, diabetic rats, and diabetic humans, have increased glycation of myofibrillar proteins (32). It remains to be seen to what extent glycation of myofibrillar proteins can be prevented with old age, but the unloaded shortening velocity of types I and Ila fibers also is reduced in master athletes. (14). Whatever the cause, this slowing of contractile properties would lead to a reduction in powergenerating capacity of the muscle tissue above that caused by the shift in fiber-type composition. It also is important to consider that the changes in fiber-type distribution and/or atrophy that underlie the age-related changes in muscle also may have implications for the stiffness of the muscle. It is known that type II fibers in animals are more compliant than type I (12), so any atrophy of type II or increased distribution of type I fibers with aging should in theory increase the muscle stiffness (which is, of course, activation modulated). The implications of increased muscle stiffness for tendon function will be considered in the next section.

Although the fast-to-slow transition in fiber-type composition and the slowing of fibers of a given type have been regularly observed, their quantitative contribution to the slowing and loss of power generation and sprint performance are not clear. It is reasonable to assume that a $6 \%$ reduction in fast fibers in the 70 - to 84 -yr age group compared with 18 - to 33 -yr olds (14) would result in an approximately $6 \%$ reduction in power. It remains to be seen to what extent the
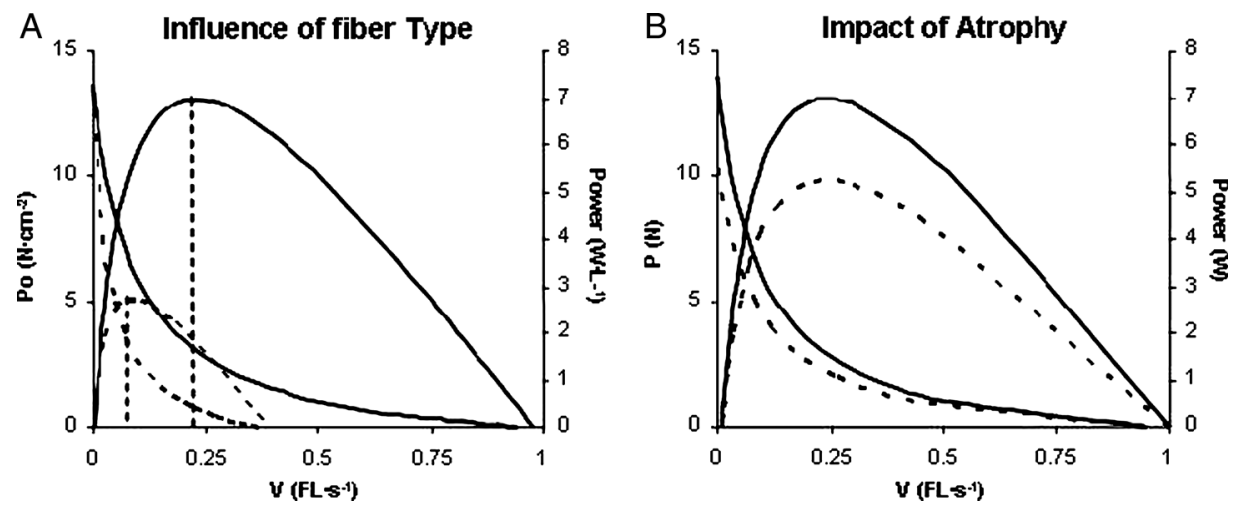

Figure 3. Normalized force-velocity and power-velocity relationships with curves following the Hill equation: $(V+b)(P+a)=b\left(P_{0}+a\right)$, where $P$ is the force the muscle produces, $V$ the velocity of shortening normalized to fiber lengths (FL) per second, $P_{0}$ the specific tension or maximal force the muscle can generate during an isometric smooth tetanus normalized by cross-sectional area, and ' $a$ ' and ' $b$ ' are constants. In general, muscle power is affected by changes in muscle volume because alterations in cross-sectional area and fiber length that determine volume are related to force and velocity capacity, respectively, that determine power. A. The fast type II fibers (-) have a higher maximal-shortening velocity and higher (in the example, 2.6-fold) power-generating capacity than the slow type III fibers (- - -). The vertical dotted lines show the velocities at which type I and type II fibers produce maximal power. $V$ indicates shortening velocity in fiber length per second; $P_{0}$, specific tension as force per fiber cross-sectional area. Data from (11). B. The solid line shows the force-velocity relationship for a hypothetical muscle with a cross-sectional area of $1 \mathrm{~cm}^{2}$. The dotted line shows the same muscle, but after $25 \%$ atrophy. The atrophy has no effect on the maximal velocity. As a result, the power-generating capacity of the muscle is reduced (by $25 \%$ ), but the velocity at which maximal power is produced is not affected. 
posttranslational modifications of the myofibrillar proteins contribute to the age-related slowing and decline in sprint performance as it is in particular the slower (types I and IIa) fibers that are affected. The obvious main factor for the loss of power and sprint performance during aging, however, is the loss of muscle mass or sarcopenia $(9,15,17)$ (Fig. 3B), which is even evident in master athletes as a reduction in average fiber cross-sectional areas (14). It has been reported that the reduction in muscle mass can be as much as $40 \%$ between 20 and $80 \mathrm{yr}$ (17), which would result in a similar reduction in maximal power-generating capacity and the resulting slowing. The latter can be understood when Figure 3B is considered; to reach similar forces (given the same activation level), the atrophied muscle has to contract at a lower speed and in this way additionally contribute to the decline in sprint performance with age. There also are other changes in muscle architecture with reductions both in pennation angle and fiber length $(23,33)$. We previously have suggested that the reduction in muscle pennation angle with atrophy would orient the fibers more along the line of pull, and thereby result in some attenuation of force loss and even a slight increase in the maximal shortening velocity of the muscle (9). The effect of a smaller pennation angle with aging, however, is expected to be quite small, as the gastrocnemius muscle's angle of pennation was only $2 \%$ different between young and old men. The main architectural factor affecting shortening velocity is the reduction in fascicle length (between $\sim 10 \%$ and $19 \%$ in the gastrocnemius of older men), explaining almost half of the loss in maximal contraction velocity $(23,33)$. However, it is important to note that fascicle length is not always reduced in elite male master sprinters (14), for example, or in elderly sedentary subjects, especially if normalized relative to limb length. Leaving these considerations and possible age-related changes in the shape of the force-velocity relationship aside, it is clear from the issues previously discussed that the reduction in power is proportional to the loss of muscle mass. Sarcopenia is, therefore, the principal contributor to the age-related loss of power-generating capacity and hence slowing.

\section{Age-Related Changes in Tendon Mechanical Properties}

Sprint performance is determined, in part, by the rates at which force can be applied to the ground (29). Although muscle strength of the leg extensor muscles is an important determinant of the ground reaction force, tendons make a profound contribution to the muscle force and power production. The compliance of the tendon affects 1) the energy storage and return from the tendon, 2) the rate of muscle force generation and transmission to the skeleton, and 3 ) the forceand power-producing capability of the muscle caused by the effects on the muscle force-length-velocity relationship because the compliant tendon is acting in series with the contracting muscle.

We have shown that, as running velocity increases, there are higher resultant moments at the ankle and knee joints (2), with moments at the ankle joint about two times higher than knee joint moments. The specific architecture of the triceps surae muscles (i.e., short fascicle length and large angle of pennation), which is more appropriate for effective force generation contributes to the high ankle joint moments.
Recent studies while running (19) report a continuous shortening of the fascicles of the gastrocnemius medialis muscle during the stance phase. During a concentric contraction, a stiffer tendon would decrease the amount and velocity of shortening of the muscle fibers for a given joint movement. A decrease in the fiber shortening velocity results in an increase of the muscle force potential caused by the force-velocity relationship. Therefore, a stiffer tendon at the triceps surae muscle-tendon unit serves to increase the rate of force transmission to the skeleton and the ground reaction force. Indeed, when we compared different groups of runners, sprinters exhibited higher tendon stiffness and contractile strength at the triceps surae muscle compared with endurance runners (3). Therefore, it can be argued that stiffer tendons and larger triceps surae muscle mass favor sprint performance. The aging process is associated with a decrease in tendon stiffness in the lower extremities and in the triceps surae muscle, for example, we have shown that stiffness is reduced by about $30 \%$ with age $(21,22)$. A less stiff Achilles tendon in combination with a reduced muscle strength capacity would compromise force generation, stride length, and sprint running performance.

On the contrary, at the quadriceps femoris muscle, a more compliant tendon was related to better sprint performance (31). The maximal elongation of the tendon and aponeurosis measured during a maximal isometric contraction correlated significantly $(r=-0.567, P=0.003)$ with the $100-\mathrm{m}$ sprint time (31). The reasons for the beneficial effects of a compliant tendon on sprinting performance in this muscle group were considered to be the increase of energy storage and return in the tendon at a given tendon force and the increase of muscle force potential as an effect of the force-velocity relationship. It has been shown that the contractile element of the quadriceps muscles experienced a stretch-shortening cycle while running (30), meaning that during the first part of the contact phase in running, the muscle-tendon unit is lengthening and so does the tendon caused by the developed forces. Consequently, strain energy is stored in the tendon. In the subsequent (second) part of the contact phase, the muscletendon unit shortens and the stored strain energy of the tendon can be reused. A more compliant tendon stretched to a greater degree by a given tendon force increases the energy storage within the muscle-tendon unit. At the second part of the contact phase (i.e., during the shortening), the increased elongation of the tendon can reduce the shortening velocity of the quadriceps femoris muscle fibers, increasing the muscle force potential caused by the force-velocity relationship. It can, therefore, be suggested that the age-related decrease in tendon stiffness at the quadriceps femoris muscle would benefit running speed. However, our work shows that, because of simultaneous age-related reduction of muscle strength, the maximal elongation of the tendon and aponeurosis is similar in the quadriceps muscle between young and old adults and the energy storage capacity of the tendon is significantly lower $(\sim 35 \%)$ in old compared with young adults (22). Therefore, the synchronous reduction in muscle strength and tendon stiffness at the quadriceps femoris muscle results instead to an actual decline in sprint performance.

In the previous section, it was explained that changes in the muscle fiber-type distribution and/or atrophy with aging may in fact increase the stiffness of the muscle. In theory, if 
the tendon stiffness was unaffected by aging, then an increased stiffness of the muscle would help storage and release of elastic energy by the tendon and the speed of the muscle-tendon unit. However, our work shows that the tendon is not unaffected, and there is normally a reduction of tendon stiffness in older people (21). This will counteract the effects of increased muscle stiffness, and it is interesting to note that the apparent vertical stiffness in older runners (which is the overall stiffness of the body affected by the active muscle-tendon units and other structures acting in series), has been shown recently to be the same as in young runners, leading to the same natural frequency of the oscillating body during running (5). It is clear, therefore, that the actual effects of the changes in tendon properties with aging on sprint performance depend very much on the interactions of muscle and tendon function and the role of the specific muscle-tendon unit and joint in the mechanics of the movement.

\section{Conclusions Regarding the Effects of Muscle and Tendon Properties on Sprinting}

From the evidence considered here and recent work on running and sprinting in older men $(5,14)$, it is evident that the three main contributors to the reduction in maximal running velocity are the lower maximum strength of lower limb muscles, the slower rate of force development and transmission, and the reduction in elastic energy storage and recovery in tendons. Although the tendons of different muscle tendon units of the lower limbs seem to have different effects depending on the contribution of the different joints to running mechanics, it is clear that the loss of muscle mass and the deterioration of muscle contractile properties are the main factors slowing down old sprinters. Fortunately, the plasticity of the neuromuscular and musculoskeletal systems allows the adaptability of neuromuscular performance parameters with highly specific training and a partial reduction in the rate of decline of the physiological characteristics associated with aging.

\section{References}

1. Andersen JL. Muscle fiber type adaptation in the elderly human muscle. Scand. J. Med. Sci. Sports 2003; 13:40-7.

2. Arampatzis A, Brüggemann GP, Metzler V. The effect of speed on leg stiffness and joint kinetics in human running. J. Biomech. 1999; 32: 1349-53.

3. Arampatzis A, Karamanidis K, Morey-Klapsing G, De Monte G, Stafilidis S. Mechanical properties of the triceps surae tendon and aponeurosis in relation to intensity of sport activity. J. Biomech. 2007; 40:1946-52.

4. Baker AB, Tang YQ, Turner MJ. Percentage decline in masters superathlete track and field performance with aging. Exp. Aging Res. 2003; 29:47-65.

5. Cavagna GA, Legramandi MA, Peyre-Tartaruga LA. Old men running: mechanical work and elastic bounce. Proc. Biol. Sci. 2008; 275:411-8.

6. D'Antona G, Pellegrino MA, Carlizzi CN, Bottinelli R. Deterioration of contractile properties of muscle fibers in elderly subjects is modulated by the level of physical activity. Eur. J. Appl. Physiol. 2007; 100:603-11.

7. Degens H, Yu F, Li X, Larsson L. Effects of age and gender on shortening velocity and myosin isoforms in single rat muscle fibers. Acta. Physiol. Scand. 1998; 163:33-40.

8. Degens $\mathrm{H}$. The role of systemic inflammation in age-related muscle weakness and wasting. Scand. J. Med. Sci. Sports 2010; 20:28-38.

9. Degens H, Erskine RM, Morse CI. Disproportionate changes in skeletal muscle strength and size with resistance training and aging. J. Musculoskelet. Neuronal Interact. 2009; 9:123-9.
10. Donato AJ, Tench K, Glueck DH, Seals DR, Eskurza I, Tanaka H. Declines in physiological functional capacity with age: a longitudinal study in peak swimming performance. J. Appl. Physiol. 2003; 94:764-9.

11. Gilliver SF, Degens H, Rittweger J, Sargeant AJ, Jones DA. Variation in the determinants of power of chemically skinned human muscle fibers. Exp. Physiol. 2009; 94:1070-8.

12. Goubel F, Marini JF. Fiber type transition and stiffness modification of soleus muscle of trained rats. Pflugers Arch. 1987; 410:321-5.

13. Hill A. The heat of shortening and the dynamic constants of muscle. Proc. R. Soc. B. Biol. Sci. 1938; 126:136-95.

14. Korhonen MT, Cristea A, Alen M, et al. Aging, muscle fiber type, and contractile function in sprint-trained athletes. J. Appl. Physiol. 2006; 101:906-17.

15. Larsson L. Morphological and functional characteristics of the aging skeletal muscle in man. A cross-sectional study. Acta Physiol. Scand. Suppl. 1978; 457:1-36.

16. Larsson L, Li X, Yu F, Degens H. Age-related changes in contractile properties and expression of myosin isoforms in single skeletal muscle cells. Muscle Nerve Suppl. 1997; 5:S74-8.

17. Lexell J, Taylor CC, Sjostrom M. What is the cause of the aging atrophy? Total number, size and proportion of different fiber types studied in whole vastus lateralis muscle from 15- to 83-year-old men. J. Neurol. Sci. 1988; 84:275-94.

18. Lexell J. Evidence for nervous system degeneration with advancing age. J. Nutr. 1997; 127:1011S-3S.

19. Lichtwark GA, Bougoulias K, Wilson AM. Muscle fascicle and series elastic element length changes along the length of the human gastrocnemius during walking and running. J. Biomech. 2007; 40:157-64.

20. Longo UG, Rittweger J, Garau G, et al. No influence of age, gender, weight, height, and impact profile in Achilles tendinopathy in masters track and field athletes. Am. J. Sports Med. 2009; 37:1400-5.

21. Mademli L, Arampatzis A. Mechanical and morphological properties of the triceps surae muscle-tendon unit in old and young adults and their interaction with a submaximal fatiguing contraction. J. Electromyogr. Kinesiol. 2008; 18:89-98.

22. Mademli L, Arampatzis A, Walsh M. Age related effect of static and cyclic loading on the strain-force curve of the vastus lateralis tendon and aponeurosis. J. Biomech. Eng. 2008; 130:011007.

23. Narici MV, Maganaris CN, Reeves ND, Capodaglio P. Effect of aging on human muscle architecture. J. Appl. Physiol. 2003; 95:2229-34.

24. Nishikawa T, Edelstein D, Du XL, et al. Normalizing mitochondrial superoxide production blocks three pathways of hyperglycaemic damage. Nature 2000; 404:787-90.

25. Pearson SJ, Young A, Macaluso A, et al. Muscle function in elite master weightlifters. Med. Sci. Sports Exerc. 2002; 34:1199-206.

26. Pollock ML, Foster C, Knapp D, Rod JL, Schmidt DH. Effect of age and training on aerobic capacity and body composition of master athletes. J. Appl. Physiol. 1987; 62:725-31.

27. Pollock ML, Mengelkoch LJ, Graves JE, et al. Twenty-year follow-up of aerobic power and body composition of older track athletes. J. Appl. Physiol. 1997; 82:1508-16.

28. Ramamurthy B, Hook P, Jones AD, Larsson L. Changes in myosin structure and function in response to glycation. FASEB J. 2001; 15:2415-22.

29. Rittweger J, di Prampero PE, Maffulli N, Narici MV. Sprint and endurance power and aging: an analysis of master athletic world records. Proc. Biol. Sci. 2009; 276:683-9.

30. Sasaki K, Neptune RR. Muscle mechanical work and elastic energy utilization during walking and running near the preferred gait transition speed. Gait Posture 2006; 23:383-90.

31. Stafilidis S, Arampatzis A. Muscle-tendon unit mechanical and morphological properties and sprint performance. J. Sports Sci. 2007; 25:1035-46.

32. Syrovy I, Hodny Z. Non-enzymatic glycosylation of myosin: effects of diabetes and aging. Gen. Physiol. Biophys. 1992; 11:301-7.

33. Thom JM, Morse CI, Birch KM, Narici MV. Influence of muscle architecture on the torque and power-velocity characteristics of young and elderly men. Eur. J. Appl. Physiol. 2007; 100:613-9.

34. Trappe S, Gallagher P, Harber M, Carrithers J, Fluckey J, Trappe T. Single muscle fiber contractile properties in young and old men and women. J. Physiol. 2003; 552:47-58. 